\title{
Análisis de las Interacciones entre Alumnado y Diversas Personas Adultas en Actuaciones Educativas de Éxito: Hacia la Inclusión de Todos y Todas
}

\section{Analysis of the Interactions between Students and Diverse Adults in Successful Educational Actions: Towards the Inclusion for All}

\author{
Rocío García Carrión 1* \\ Silvia Molina Roldán ${ }^{2}$ \\ Luis Antonio Grande López ${ }^{3}$ \\ Nataly Buslón Valdez ${ }^{4}$ \\ ${ }^{1}$ Universidad de Deusto ${ }^{2}$ Universitat Rovira i Virgili \\ ${ }^{3}$ Centro de Profesorado de Jerez de la Frontera ${ }^{4}$ Universidad de Barcelona
}

\begin{abstract}
Más de 200 escuelas en Europa y más de 400 en Latinoamérica están implementando Actuaciones Educativas de Éxito (AEE) que están contribuyendo a la mejora educativa de alumnado muy diverso. Entre esta diversidad encontramos al alumnado con dificultades de aprendizaje y con discapacidad. En este artículo revisamos las aportaciones que dos AEE, los Grupos Interactivos y las Tertulias Literarias Dialógicas, están haciendo a la mejora del aprendizaje instrumental y social de todo el alumnado, y especialmente de aquellos con más dificultades. Nos centramos en el tipo de interacciones que estas AEE promueven entre el alumnado, con el profesorado y con otros adultos de la comunidad, en el impacto que estas interacciones tienen en su aprendizaje y participación en el grupo, para mostrar cómo estas AEE contribuyen a una educación inclusiva.
\end{abstract}

Descriptores: Aprendizaje, Interacción, Comunidad, Alumnado con necesidades especiales.

\begin{abstract}
More than two hundred schools in Europe and more than four hundred schools in Latin American are implementing Successful Educational Actions (SEAs) that are contributing to academic success of diverse students. Among this diversity we find pupils with learning difficulties and with disabilities. In this article, we review the contributions that two SEAs -Interactive Groups and Dialogic Literary Gatherings- are making to improve academic and social learning for all students, and especially for those with more difficulties. We focus on the type of interactions that these SEAs promote among pupils, with teachers and other adults from the community. Particularly, our emphasis relies on the impact that these interactions have on their learning and on their participation within the group, to show how these SEAs contribute to an inclusive education.
\end{abstract}

Keywords: Learning, Interaction, Community, Special needs students.

\footnotetext{
*Contacto: rocio.garcia@deusto.es
} 


\section{Conceptualizando el papel clave de la interacción y el diálogo en el aprendizaje}

Los avances de la investigación en los campos de la psicología y la educación cada vez ponen más de manifiesto el papel clave que tienen la interacción y el diálogo en el aprendizaje, enfoque que se ha conceptualizado como el giro dialógico en la comprensión de los procesos de aprendizaje (Racionero y Padrós, 2010). Tal conceptualización se ha desarrollado sobre la base de la teoría sociocultural del aprendizaje, cuyos fundamentos residen en las aportaciones de Vygotsky (1962, 1979), según la cual el aprendizaje y el desarrollo cognitivo son procesos culturales que se hacen posible en diálogo e interacción con los otros (Cole, 1996; Rogoff, 1990; Wenger, 2001). Estas teorías son las que mejor explican actualmente el aprendizaje y que mejor responden al reto de conseguir el éxito educativo para todos y todas.

Si la naturaleza del aprendizaje humano es eminentemente social, es decir, tiene lugar en contextos compartidos y en interacción con otras personas, comprender cómo se da el aprendizaje pasa por comprender esas interacciones. Las implicaciones de esta concepción social de la cognición son profundamente transformadoras, puesto que, en el plano social podemos transformar las interacciones y, por tanto, el contexto, para crear las máximas oportunidades de aprendizaje para todas y todos (Vygotsky, 1979). Las interacciones entre iguales han mostrado efectos positivos en el progreso en el aprendizaje del alumnado más vulnerable (Durán y Valdebenito, 2014), y éstos se incrementan con la implicación de las familias y otras personas de la comunidad dentro y fuera de la escuela (García-Carrión y Díez Palomar, 2015). De esta manera, una concepción interactiva del aprendizaje supera las concepciones constructivistas que obvian los procesos de construcción conjunta de conocimiento y que, centradas en la construcción individual del mismo, y bajo el principio de la adaptación a la diversidad, ha dado lugar a prácticas pedagógicas que han demostrado no ser efectivas (Clark y Estes, 1998, 1999; Estes y Clark, 1999; Kirschner, Martens, y Strijbos, 2004). Estas prácticas, además, han aumentado las desigualdades entre grupos de alumnos con diferentes niveles previos de aprendizaje, frecuentemente reproduciendo las desigualdades sociales y culturales (Oakes, 2005). Bruner, cuya teoría nació ligada a perspectivas más internalistas del constructivismo y al procesamiento de la información, critica la revolución cognitiva que él mismo lideró hace más de cuatro décadas y enfatiza la intersubjetividad como clave para entender los procesos mentales humanos (Bruner, 1996). En la actualidad, ante la cuestión de qué debería estudiar la psicología, insiste en la creación de nuevos mundos posibles que se crean de forma intersubjetiva (Bruner, 2012). El alumnado con dificultades de aprendizaje más importantes y con discapacidades también se beneficia del potencial de la intersubjetividad para avanzar a estados superiores del desarrollo; en palabras de Vygotsky en relación a este alumnado "la escuela debería esforzarse en ayudarles en este sentido y en desarrollar en su interior aquello de lo que carecen intrínsecamente en su desarrollo" (Vygotsky, 1979, p. 137).

No sólo el profesorado sino también familiares y miembros de la comunidad pueden hacer uso de la principal herramienta cultural y psicológica que vehicula el pensamiento: el lenguaje (Vygotsky, 1962), y así crear conocimiento entre personas que dialogan (Edwards y Mercer, 1987; Flecha, 1997; Wells, 1999). El lenguaje juega un papel esencial tanto como herramienta para el pensamiento como para mediar la actividad, y tanto en el plano social como en el psicológico. El diálogo en la interacción con los otros 
permite compartir el foco de atención y los propósitos (Rogoff, 1990), facilita la comprensión de la mente de otras personas (Bruner, 1997) y hace posible alcanzar acuerdos (Habermas, 1987). Además esos diálogos que se internalizan, generan un aprendizaje y comprensión más profunda a través de la cadena de diálogos e interacciones que se establecen dentro de la misma persona (Bakhtin, 1981). Si consideramos que cada emisión lingüística o afirmación producida por cada participante en una situación de diálogo es un eslabón en una cadena de interacciones dialógicas, cada nueva emisión se construye en función de las anteriores, y de alguna manera las incluye. Cuantas más interacciones comunicativas tengamos y de más calidad, más incorporaremos estas aportaciones a las nuestras propias, más enriqueceremos nuestras reflexiones.

Décadas de investigación sobre el habla y el diálogo en el aula han demostrado que la calidad del diálogo en el discurso del aula tiene un fuerte impacto en aspectos tales como las habilidades de lectoescritura y comprensión lectora del alumnado (Howe y Abedin, 2013; Mercer y Littleton, 2007; Nystrand, 2006; Resnick, Asterhan y Clarke, 2015). Sin embargo la revisión de la literatura en este campo indica que raramente el alumnado tiene oportunidades de participar en conversaciones dialógicas en la mayoría de aulas (Nystrand y Gamoran, 1991; Nystrand, Gamoran, y Heck, 1993; Nystrand, Gamoran, Kachur y Prendergast, 1997). Con frecuencia la participación del alumnado en el diálogo consiste en recordar hechos (memoria factual) o reportar el conocimiento de otros más que el de uno mismo. Trabajos como los de Alexander (2001), Mercer (2000), Wells (1999), Anderson y colaboradores (1998) y Flecha (1997) han avanzado en identificar qué condiciones tienen que reunir las interacciones dialógicas para que consigan los máximos niveles de desarrollo cognitivo, social y emocional, y han conceptualizado formas concretas que maximizan el aprendizaje a través del diálogo.

Alexander (2001) destaca la importancia de la calidad del diálogo en el aula para promover el aprendizaje, y define la "enseñanza dialógica" (dialogic teaching) como el diálogo efectivo en el aula que se caracteriza por cinco principios: es colectivo, recíproco, proporciona apoyo, es acumulativo y tiene un propósito (Alexander, 2008), principios que enfatizan la horizontalidad del diálogo y la creación conjunta de conocimiento. Wells (1999) describe cómo a través de interacciones dialógicas los participantes construyen significados a partir de una secuencia de emisiones lingüísticas encadenadas, que incluyen preguntas y respuestas para dar solución a una situación de indagación, y cómo se pueden crear en el aula comunidades de indagación dialógica que potencien estas interacciones, que llevan también a la elaboración conjunta de conocimiento.

Mercer (2000) entiende el diálogo como un "modelo social de pensamiento" que hace posible un proceso denominado "interpensar", en el que los participantes pueden resolver problemas y co-construir su aprendizaje conjuntamente. Cuando en las interacciones entre el alumnado éstos se implican de manera crítica y constructiva en las ideas aportadas por los otros, se da un "habla exploratoria", donde las ideas pueden ser cuestionadas a través de la argumentación, con la finalidad de alcanzar acuerdos y progresar en la comprensión. Interacciones dialógicas de este tipo entre alumnado (Mercer y Littleton, 2007) y entre alumnado y profesorado (Rojas-Drummond y Mercer, 2003) han mostrado influir positivamente en el aprendizaje de matemáticas, lengua y ciencias. 
Por otro lado, el razonamiento colaborativo (collaborative reasoning) propuesto por Anderson, Chinn, Waggoner y Nguyen (1998) es un enfoque de enseñanza que promueve la socialización del pensamiento y la calidad del habla en el aula. Consiste en crear un foro de discusión libre guiado por el propio alumnado y con la mínima intervención del profesorado con la intención de guiar una argumentación auténtica entre grupos de alumnado heterogéneo para encontrar la solución a un problema. Este tipo de práctica se ha aplicado con el objetivo de mejorar las habilidades de lectura y de razonamiento del alumnado y ha demostrado promover la adquisición de habilidades de argumentación.

La teoría del aprendizaje dialógico, desarrollada primero en relación al aprendizaje de personas adultas (Flecha, 1997) y más tarde en el aprendizaje en edad escolar (Aubert, Flecha, García, Flecha y Racionero, 2008), expone que lo que mejor explica el aprendizaje no es sólo la oportunidad de dialogar, sino el hecho de hacerlo entre personas diversas, incluyendo alumnado, profesorado, familiares y otras personas de la comunidad con diferentes conocimientos, experiencias y culturas. Para que el aprendizaje sea dialógico ha de basarse en siete principios: diálogo igualitario, dimensión instrumental, inteligencia cultural, transformación, creación de sentido, solidaridad e igualdad de diferencias. El aprendizaje organizado alrededor de estos siete principios consigue mejorar tanto el rendimiento académico como el clima de convivencia en los centros educativos y las comunidades en que se insertan (Racionero, Ortega, García y Flecha, 2012).

Los autores y teorías revisados nos muestran la posibilidad de crear entornos más ricos de aprendizaje y que promueven un mejor desarrollo cognitivo cuando se hace un uso efectivo del diálogo. En base a estas aportaciones, las aulas escolares deberían organizarse cada vez más con el objetivo de proporcionar un ambiente interactivo en el que el alumnado pueda dialogar, intercambiar ideas y compartir conocimiento. En la medida en que la práctica escolar se ajuste a estas evidencias estaremos promoviendo intervenciones educativas que respondan a una concepción científica del aprendizaje y el desarrollo.

En base a estas aportaciones, en este artículo analizamos el tipo de interacciones que se establecen en dos Actuaciones Educativas de Éxito (AEE): Grupos Interactivos y Tertulias Literarias Dialógicas. Si bien sabemos que estas AEE están consiguiendo el éxito educativo de alumnado diverso, conocemos menos los procesos concretos de interacción y diálogo que favorecen la creación de aulas más dialógicas y a la vez más inclusivas. Nos centramos, por tanto, en el análisis de la interacción que puede generar más oportunidades de aprendizaje para todos y todas, incluyendo el alumnado con necesidades especiales. A continuación describimos el marco donde se ha llevado a cabo la investigación, definimos el concepto de Actuaciones Educativas de Éxito y describimos el contexto de aula en el que hemos realizado en análisis de las interacciones: Grupos Interactivos y las Tertulias Literarias Dialógicas. 


\section{Actuaciones educativas de éxito: creando aulas inclusivas en grupos interactivos y tertulias literarias dialógicas}

Las Actuaciones Educativas de Éxito (AEE) emergen como uno de los principales resultados de la única investigación en ciencias socioeconómicas y humanidades incluida en el listado la Comisión Europea de 10 ejemplos de investigaciones de éxito en Europa. Esta investigación fue INCLUD-ED. Strategies for inclusion and social cohesion in Europe from education desarrollado entre 2006 y 2011 dentro del $6^{\circ}$ Programa Marco de la Comisión Europea. Su principal objetivo fue identificar las actuaciones educativas que llevan al éxito escolar, superan desigualdades y promueven la cohesión social, presentando especial atención a grupos vulnerables como las minorías culturales y las personas con discapacidad. En el transcurso del proyecto se realizó un análisis comprensivo que incluyó políticas educativas, resultados, y prácticas, así como el análisis de centros educativos de éxito.

Entre sus principales resultados, se identificaron, analizaron y definieron las AEE; actuaciones que han demostrado conseguir los mejores resultados en los diferentes contextos donde se han aplicado, por tanto, contienen elementos que son universales y en consecuencia transferibles a otros contextos educativos y sociales. Concretamente, en estos diferentes contextos (geográficos, económicos, sociales o culturales) las AEE han logrado reducir el absentismo y el abandono escolar temprano, aumentar los resultados académicos, generar altas expectativas para todo el alumnado, y mejorar la convivencia y los valores. Como consecuencia, hoy las AEE se aplican en más de 200 centros en España y más de 400 en otros países como Brasil, Chile, Perú, México, Guatemala, Inglaterra o Argentina y la Comisión Europea ha recomendado el desarrollo de escuelas como comunidades de aprendizaje como estrategia para reducir el abandono escolar temprano (European Commission., 2011), destacando especialmente su aportación a la inclusión del alumnado con discapacidades (European Council., 2010).

La organización de los contextos de aprendizaje que constituyen las AEE se basan en dos criterios esenciales. En primer lugar, el alumnado se agrupa siempre siguiendo el criterio de heterogeneidad, evitando cualquier tipo de segregación por niveles, separación del grupo natural para trabajar contenidos diferenciados, o adaptaciones acríticas del currículo que rebajan las expectativas y oportunidades de aprendizaje, y potenciando la riqueza de interacciones que se hace posible en esa diversidad (Molina y Ríos, 2010). En segundo lugar, los recursos disponibles para apoyar el aprendizaje del alumnado se organizan dentro de estos grupos heterogéneos. La apertura a distintos agentes en las aulas, facilita no sólo la reducción de las ratios, sino la creación de nuevos espacios de diálogo, intercambio, cooperación y solidaridad entre el alumnado y con personas adultas (Herrero y Brown, 2010), que refuerza e impulsa los procesos de aprendizaje de todos los niños y las niñas, y especialmente de aquellos con un nivel de aprendizaje más bajo o que presenta mayores dificultades.

En este sentido, las AEE son una herramienta eficaz de participación y trabajo coordinado entre distintos agentes de la comunidad -maestros, familias, voluntarios, entre otros- que permiten una organización de los espacios e interacciones de aprendizaje encaminados a conseguir aprendizajes de máximos. De esta manera, las AEE superan las prácticas que tienden a agrupar al alumnado por niveles, creando 
oportunidades de aprendizaje desiguales, que refuerzan desigualdades previas. En la medida en que ofrecen una educación igualitaria, de altas expectativas y de interacciones ricas en oportunidades de aprendizaje para todos y todas, las comunidades de aprendizaje desarrollan un modelo de escuela inclusiva.

Si bien existe un consenso plasmado en declaraciones internacionales sobre el derecho universal a una educación inclusiva, y la importancia de ésta para conseguir la cohesión social (UNESCO., 1990; 1994), aun continúa siendo un reto hacer realidad este derecho. Alumnado con dificultades de aprendizaje y con contextos desfavorecidos tienden a encontrar barreras al aprendizaje y la participación (Booth y Ainscow, 2002) en un sistema educativo que aplica prácticas de segregación y adaptaciones curriculares que enfatizan las dificultades individuales y tienen consecuencias exclusoras, impactando negativamente en las posibilidades de aprendizaje de este alumnado y su la inclusión social futura (Ainscow et al., 2006). Las AEE son una respuesta ante la pregunta de cómo llevar a la práctica la inclusión, en términos de asistencia, rendimiento y participación de todos y todas, teniendo en cuenta las comunidades donde las comunidades se insertan (Ainscow, 2003; Ainscow et al., 2012). Contemplan la heterogeneidad, en términos culturales, étnicos, de género, o de capacidades, en vista de la aplicación del principio de igualdad de diferencias (Flecha, 1997), desde el cual las distintas identidades son respetadas y valoradas: desde todas ellas se puede avanzar en el aprendizaje y todas ellas son un recurso valioso para el aprendizaje de los demás.

Los Grupos Interactivos y las Tertulias literarias dialógicas son dos de las AEE que mejores resultados están obteniendo en la mejora educativa de alumnado diverso. Los Grupos Interactivos (GI) (Valls y Kyriakides, 2013) consisten en dividir la clase en pequeños grupos heterogéneos de alumnado, generalmente para trabajar en materias instrumentales con la ayuda de personas voluntarias que dinamizan las interacciones entre el alumnado. Los GI promueven la ayuda mutua a través del principio de solidaridad, reflejando el esquema de las sub-comunidades de aprendices mutuos (Bruner, 1997); en este sentido, la diversidad entre los alumnos se entiende como un factor positivo para el aprendizaje y en consecuencia todos los estudiantes encuentran el apoyo que necesitan realizando actividades con otras personas que tienen habilidades distintas a las suyas. Al incluir la participación del voluntariado, ex/alumnos, familiares y personas de la comunidad, los GI logran multiplicar y diversificar las interacciones, a la vez que aumenta el tiempo de trabajo efectivo. De esta manera, los GI evitan la segregación del alumnado de acuerdo a sus niveles de aprendizaje o sus capacidades, que la investigación ha asociado con bajos resultados académicos, baja autoestima y expectativas (Dunn, 1968; Fitch, 2003, Fisher, Roach, Frey, 2002), y reducen la aplicación de adaptaciones curriculares. Por el contrario, los GI responden a las distintas necesidades de los estudiantes y sus dificultades, mediante la reorganización de recursos existentes en la comunidad.

Las Tertulias Literarias Dialógicas (TLD) consisten en debatir la lectura de obras de la literatura clásica universal. Durante esta actividad el alumnado comparte las ideas sugeridas por la lectura en un espacio de diálogo donde se reflexiona sobre lo leído. La creación de significados, la comprensión y el aprendizaje se intensifican por medio de las interacciones que se crean entre los participantes (Aguilar et al., 2010). Se consigue de esta forma una interpretación interactiva del texto que, partiendo de actos comunicativos dialógicos, basados en la argumentación, otorga el mismo valor a las aportaciones emitidas por todos los participantes, independientemente de su nivel 
académico y del rol que desempeñan (Pulido y Zepa, 2010). En las TLD el alumnado encuentra un momento para conocerse mejor, definir sus opiniones y sentimientos así como para aprender conjuntamente respetando todas las voces, y se consigue que sean más valorados estudiantes que no lo son tanto en otros momentos (Molina, 2015). A través tanto de las lecturas, que tratan temas universales y atemporales, como de la propia dinámica de la tertulia, se trabajan valores como la solidaridad, la justicia, el cuidado y la responsabilidad, mientras se progresa en comprensión lectora y expresión oral.

Partiendo del conocimiento existente acerca de los beneficios que han demostrado generar las AEE que acabamos de exponer, este artículo tiene como objetivo analizar las interacciones que se establecen en los Grupos Interactivos y las Tertulias Literarias Dialógicas, para en último término identificar que tipo de interacción puede generar más oportunidades de aprendizaje para todos y todas, incluyendo el alumnado con necesidades especiales.

\section{Metodología}

Con el objetivo de analizar cuál es el tipo de interacciones que se establecen en las dos AEE que situamos como objeto de estudio: Grupos Interactivos y Tertulias Literarias Dialógicas y, en concreto, cómo éstas contribuyen a crear contextos inclusivos de aprendizaje, hemos recogido y analizado datos de diferentes escuelas que aplican estas AEE y que incluyen a alumnado con necesidades especiales. La metodología de investigación utilizada ha sido la Metodología Comunicativa (Gómez, Puigvert y Flecha, 2011), que tiene como objetivo investigar no para describir sino para contribuir desde la investigación a la inclusión de los más desfavorecidos. Para ello, uno de los elementos distintivos de esta metodología es la ruptura con el desnivel metodológico entre personas investigadoras y destinatarias de la investigación, y la incorporación del dialogo igualitario entre ambas durante el proceso de investigación. En este proceso cada persona aporta el conocimiento que le es propio: mientras las personas investigadoras proveen el conocimiento científico, los destinatarios de la investigación proporcionan el conocimiento de la experiencia vivida, y en el diálogo intersubjetivo entre ambos se genera el conocimiento resultado de la investigación.

\subsection{Recogida de datos}

Respecto a los Grupos Interactivos, hemos recogido la información de una escuela que aplica esta AEE, y nos hemos centrado en dos casos de alumnado con discapacidad motriz, uno de ellos con un retraso importante en el aprendizaje. Para ello hemos realizado, por cada caso, una observación de aula, un grupo de discusión con alumnado, una entrevista semiestructurada con orientación comunicativa con el profesorado tutor y una entrevista con una persona voluntaria en grupos interactivos. A través de las entrevistas obtuvimos también información adicional sobre otros casos de la escuela, que en este caso es una escuela situada en un barrio con un nivel socioeconómico medio. Para analizar las Tertulias Literarias Dialógicas hemos recogido la información en una segunda escuela, en este caso situada en un barrio socialmente desfavorecido y con una alta proporción de alumnado inmigrante. Nos centramos para ello en el caso de una niña con discapacidad intelectual, sobre el que realizamos un relato de vida comunicativo con 
la propia niña una entrevista en profundidad de orientación comunicativa con una de sus profesoras.

Asimismo, contamos para el análisis con información sobre GI y TLD recogida en 5 centros educativos ubicados en zonas diversas: desde zonas rurales en pedanías de grandes localidades, donde se da un alto índice de marginalidad, hasta centros inmersos en zonas urbanas de grandes poblaciones con gran riesgo de deprivación socio-cultural. En el conjunto de estos centros recogimos datos durante 24 meses con la participación de profesorado, voluntariado y familias de 9 unidades de Educación Infantil y Primaria. En concreto realizamos, por un lado, observaciones comunicativas de sesiones de GI y TLD, donde se discutió con voluntariado, familias y profesorado la influencia de estas actuaciones en el alumnado con NEE. Por otro lado, se realizaron entrevistas semistructuradas de orientación comunicativa con alumnado con necesidades educativas especiales, con familiares y con profesorado, todas ellas dirigidas a recoger cuestiones relativas al trabajo en GI y TLD y los cambios percibidos en el alumnado relación al trabajo en el marco de estas actuaciones.

\subsection{Análisis de datos}

Los datos recogidos han sido analizados desde el punto de vista de las interacciones que tienen lugar en estas AEE y que favorecen la inclusión de alumnado diverso, especialmente cuando participan en ellas alumnado con necesidades especiales. Con este objetivo se ha elaborado un cuadro de análisis formado por 3 categorías principales que se refieren a tres tipos diferentes de consecuencias que tienen en el aprendizaje las interacciones observadas:

- Interacciones que compensan y/o ayudan: incluimos aquí todas las interacciones que compensan dificultades específicas en la comprensión del contenido y/o en la realización de la actividad y que ayudan en la realización de la misma y en el aprendizaje.

- Interacciones que abren nuevas oportunidades de aprendizaje: nos referimos aquí a las interacciones que hacen posible que el alumnado participe activamente de actividades o situaciones de aprendizaje que no se darían fuera de estas AEE.

- Interacciones que amplían la participación activa y creativa del alumnado con necesidades especiales: en esta categoría encajan las interacciones que permiten no sólo avanzar en el propio aprendizaje sino contribuir al aprendizaje de los demás.

Estas categorías han sido creadas principalmente a través de un análisis inductivo de los datos (Creswell, 2002) guiado por el objetivo de identificar tipos de interacciones en el proceso de aprendizaje que facilitasen la participación del alumnado con dificultades y mostrasen un uso productivo del habla y el diálogo por su influencia positiva en el aprendizaje (Resnick, Asterhan, y Clarke, 2015). A partir de una primera revisión e interpretación de los datos recogidos, se definieron tres tipos de interacciones (ver Tabla 1), en las que se han tenido en cuenta, de manera transversal, quienes son los participantes de la interacción, pudiendo ser interacciones entre pares o con adultos (profesorado o voluntariado), así como otras características de las interacciones, tales como el diálogo igualitario o las altas expectativas, obteniendo un total de 9 categorías de análisis. 


\begin{tabular}{|c|c|c|c|}
\hline & \multicolumn{3}{|c|}{ PARTICIPANTES EN LA INTERACCIÓN } \\
\hline & \multirow{2}{*}{ Interacción entre iguales } & \multicolumn{2}{|c|}{ Interacción con adultos } \\
\hline & & Profesorado & Voluntariado \\
\hline & \multicolumn{3}{|c|}{ Diálogo igualitario, Altas expectativas, etc. } \\
\hline $\begin{array}{l}\text { Interacciones que superan dificultades } \\
\text { en el aprendizaje }\end{array}$ & $x_{5}+5$ & 4 & 7 \\
\hline $\begin{array}{l}\text { Interacciones que abren nuevas } \\
\text { oportunidades de aprendizaje }\end{array}$ & 2 & 5 & 8 \\
\hline 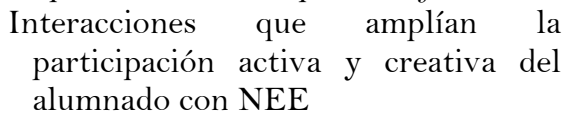 & 3 & 6 & 9 \\
\hline
\end{tabular}

Fuente: Elaboración propia.

\section{Interacciones que favorecen la inclusión a través de GI y TLD}

\subsection{Interacciones que superan dificultades en el aprendizaje}

Los GI ofrecen la posibilidad de que madres, padres, vecinos, profesorado de apoyo, estudiantes universitarios y otras personas accedan al aula y participen activamente de los procesos de aprendizaje del alumnado. De esta manera, generan nuevos formatos de interacción y diversifican las interacciones de aprendizaje. A través de la diversidad de personas adultas en el aula, se multiplican y diversifican también las posibles interacciones de ayuda y solidaridad durante el proceso de enseñanza y aprendizaje. De acuerdo a las observaciones de aula recogidas, predominan en los diferentes Grupos Interactivos interacciones de ayuda mutua entre el alumnado, y especialmente, destaca cómo esta ayuda se dirige también al alumnado que tiene más dificultades de aprendizaje. Ilustra este tipo de interacciones el caso de un aula mixta con alumnado de $5^{\mathrm{o}}$ y $6^{\mathrm{o}}$ de primaria de un centro ubicado en una zona socialmente compleja. La primera sesión de GI se llevó a cabo en el área de matemáticas durante 1:30 minutos. Durante todo el tiempo las interacciones entre el alumnado se centraron en la resolución de las tareas sin manifestar ningún tipo de enfrentamiento o conflicto entre ellos. Lejos de platearse desde una perspectiva del déficit, el nivel de las actividades era superior, incrementando así el reto cognitivo. Tras la sesión, las madres voluntarias que habían dinamizado la interacción y el diálogo en los pequeños grupos manifestaban como “increíble la manera en que se habían ayudado y respetado" (madre voluntaria).

Lo que es especialmente importante, es que el propio alumnado asume este tipo de interacciones de ayuda y solidarias con naturalidad, tal como hemos podido observar en otra de las aulas organizadas en GI en $5^{\circ}$ de primaria. Es uno de los casos de alumnado con un excelente nivel académico, que tras observar cómo una compañera de su grupo con NEE muestra dificultades para realizar la tarea pide a la voluntaria cambiarse de sitio para acompañar y ayudar a esta niña. La naturalidad con la que se asumen este tipo de interacciones permite que la ayuda a quienes más lo necesitan pueda estar integrada en el propio funcionamiento del grupo y generalizarse a otras situaciones, como la manera habitual de relacionarse. Este tipo de interacciones conforman un contexto de apoyo donde en diferentes momentos cada niño y cada niña no cuenta sólo con sus capacidades individuales, sino también con las de los demás, que les pueden ser prestadas en cualquier momento (Pea, 1993; Wertsch et al., 1995). La investigación en aprendizaje 
interactivo llevada a cabo en el Reino Unido durante más de cuatro décadas (Galton et al., 1999) ha destacado como un comportamiento propio de la enseñanza y aprendizaje interactivos la "búsqueda de ayuda" (seeking help). Este comportamiento aparece cuando el alumnado trabaja en grupo, especialmente si se incorporan los principios de una pedagogía social (Blatchford, Kutnick, Baines, y Galton, 2003). En el caso de los contextos de aprendizaje dialógico, como GI, observamos un comportamiento que va más allá de la búsqueda de ayuda, se trata de un ofrecimiento de ayuda espontánea, de altruismo, orientado a que el aprendizaje esté al alcance de todo el alumnado.

Asimismo, los GI proporcionan un espacio de interacción con miembros de la comunidad y personas adultas que no pertenecen al ámbito escolar, que ayudan al alumnado con las actividades de la clase. Su intervención con el alumnado con más dificultades va desde la ayuda puntual en algunos momentos a intervenir para ayudarles a centrar su atención y, de manera más importante, potenciar las interacciones, alentando la participación, y el apoyo mutuo dentro de cada grupo. Este tipo de interacciones se ve ejemplificado en una de las entrevistas con voluntariado, donde una de las voluntarias explica tanto ese rol de potenciar la ayuda mutua cuando no aparece espontáneamente como la naturalidad con la que los alumnos y alumnas la asumen: "Hoy no ha necesitado mucha ayuda. (...) Que si no sí que dices, 'venga, uno de los que ha terminado, que se vaya con Inés y le vaya explicando', y bueno, están encantados”. El voluntariado se convierte así en una pieza clave en la normalización y generalización de estas interacciones de ayuda.

Los GI hacen también replantear la función del profesorado de apoyo en la escuela. En los centros analizados se ha identificado un cambio en la cultura de centro y de la actitud de los profesores hacia la inclusión, cuestión que es necesario abordar desde la etapa de formación inicial del profesorado (Flores y Villardón, 2015; López, Echeita y Martin, 2009). En el caso de GI se prioriza que los apoyos específicos que un alumno o alumna necesite se ofrezcan dentro del aula durante la sesión para aprovechar al máximo las posibilidades de aprendizaje que se dan en ellos. Maestras de educación especial pueden trabajar con alumnado con discapacidades dando el respaldo y la ayuda necesaria dentro de un espacio de trabajo conjunto, sin necesidad de separar al niño o niña del grupo. Así, el centro puede reorganizar los horarios del profesorado para hacer que coincidan con GI. Es necesario destacar, no obstante, que en ocasiones estas ayudas específicas que se introducen dejan de ser necesarias mientras este alumnado está en GI. Las interacciones de ayuda con los pares y con el voluntariado transforman las dificultades de este alumnado en oportunidades reales de participación donde logran entender la actividad, resolver dudas y, en definitiva, aprovechar al máximo la sesión. Así, lo explicaba una maestra de educación especial en uno de los centros estudiados, en relación a una alumna con la que tenía muchas horas de refuerzo fuera del aula:

En el caso de esta alumna fue muy curioso porque además yo tenía muchas horas de apoyo con ella y fuera del aula (...) entonces (...), en lugar de sacarla en aquella sesión que hacen grupos interactivos, ¿̇por qué no entras con ella, dentro de clase? $\Upsilon$ claro, es que fue un cambio... un cambio total. Porque ella veía que podía hacer cosas y también los otros veían que ella las podía hacer, no (...) no era la tonta de decir "es que no sabe hacer nada", ¿̇no? y hubo un punto que casi mi figura ya tuvo que desaparecer porque los otros compañeros del grupo y con la persona voluntaria... yo poco a poco me fui retirando. 
En TLD también observamos este tipo de interacciones que ofrecen ayuda al aprendizaje. Una de las actividades que requiere participar en una tertulia es elegir al menos una frase o párrafo del libro y preparar una intervención explicando por qué esa idea es interesante. En el caso de una niña con discapacidad intelectual, cuyo caso analizamos, que tenía un nivel de lectura entre 4 y 5 cursos inferior al de sus compañeros y compañeras, se mostraba incapaz de preparar esta intervención individualmente, ya que el nivel de complejidad de la lectura y de la tarea sobrepasaba su nivel de competencia lectora. Sin embargo, cuando esta tarea de lectura y de preparación de la intervención era compartida con una persona adulta o con un compañero o compañera, la niña era capaz de realizarla con éxito. Como ella misma explicaba, estas personas "a veces me ayudan a escribir y a veces a pensar". Siguiendo las indicaciones de su compañera revisaba un párrafo, seleccionaba una idea y escribía lo que significa para ella. Además, mientras pensaba en el sentido de esa idea con su compañera, se daba cuenta de que podía conectarla con otros conceptos o situaciones que habían trabajado en otros libros, estableciendo, por ejemplo, comparaciones entre La Eneida y Don Quijote. Como resultado de estas interacciones de ayuda que andamian (Bruner, 1997) la comprensión y el razonamiento, esta niña ha conseguido hacer un gran avance tanto en comprensión lectora como en expresión oral, ambas capacidades que se trabajan intensivamente en las tertulias.

\subsection{Interacciones que abren nuevas oportunidades de aprendizaje}

GI y TLD no sólo contribuyen a apoyar el aprendizaje o la comprensión en actividades concretas, sino que crean las oportunidades para que se den nuevos aprendizajes o se desarrollen nuevas capacidades. Observamos el caso de un alumno con NEE, concretamente con grandes dificultades en la expresión escrita. Tras un trimestre sin lograr avanzar en la producción escrita, el trabajo en GI incrementó a la autoconfianza de este alumno que por primera vez en su proceso de aprendizaje de la lectoescritura fue capaz de escribir un texto con coherencia. La importancia de esta situación va más allá de la realización de esa actividad en concreto, porque implica un logro, algo que, aunque con ayuda, desde ese momento es capaz de hacer. Ayudas como éstas proporcionadas en grupos interactivos por iguales o por adultos facilitaron que un alumno de $4^{\circ}$ de primaria con un gran desfase curricular avanzase más allá de lo establecido para él en ese curso, o que una alumna de $3^{\circ}$ de primaria que no conseguía aprobar o lo hacía muy justa empezara a sacar notables y sobresalientes.

En las TLD hemos podido observar avances similares respecto de la lectura. La alumna mencionada anteriormente empezó a participar de las tertulias cuando aún no sabía leer. En este contexto pudo, por un lado, enriquecerse de los debates que sus compañeros y compañeras tenían alrededor del texto; de esta manera, aunque ella no podía acceder individualmente al contenido del libro, sí que lo podía hacer a través de la lectura que habían hecho sus compañeros de clase. Por otro lado, esta posibilidad de conocer a través de sus compañeros lo que los libros explicaban hizo crecer en ella una gran motivación por aprender a leer. De esta manera, las TLD muestran que el alumnado es capaz de aprovechar una situación de aprendizaje que está más allá de sus capacidades individuales en un momento dado, porque puede apoyarse en las capacidades de otras personas $\mathrm{y}$, a través de interacciones compartidas, desarrollar estas capacidades en su interior (Vygotsky, 1979). Ya en sexto curso de primaria, esta niña es capaz de leer, aunque su nivel continúa siendo marcadamente inferior al de los niños y niñas de su edad. Individualmente es capaz de leer sólo libros del nivel de primero o segundo de 
primaria, sin embargo, en las TLD lee los mismos libros que sus compañeros y compañeras y los debate con ellos y ellas. Este caso nos muestra cómo las interacciones que tienen lugar en esta AEE permiten alcanzar nuevos hitos -como aprender a leer o leer libros de un nivel acorde a la edad- que no se alcanzan fuera de este contexto de aprendizaje.

Estos resultados nos muestran que no sólo las expectativas influyen en el aprendizaje sino también al revés, las expectativas hacia este alumnado se transforman cuando se les ofrecen oportunidades más ricas de aprendizaje porque tienen más apoyos disponibles y participan de más interacciones de aprendizaje. Lo que parecía imposible se hace posible, y eso cambia la autoimagen y autoconcepto del alumnado, así como la imagen y concepto que los demás, iguales y adultos, tienen de ellos, y que tiene a su vez tanta importancia en el aprendizaje. La apertura de nuevas oportunidades de aprendizaje que tiene lugar en GI y TLD supone, así, una alternativa a la práctica de las adaptaciones curriculares, especialmente cuando, centradas exclusivamente en las capacidades que un alumno muestra en un momento dado, constituyen adaptaciones exclusoras (INCLUD-ED Consortium, 2011) que limitan las oportunidades de aprendizaje. La atención a las necesidades especiales desde las AEE toma de referencia no sólo al individuo sino también el contexto donde éste aprende y se desarrolla. Esta perspectiva es la que permite responder de manera más efectiva a las necesidades especiales del alumnado, aumentar las expectativas y evitar la reducción del currículum.

\subsection{Interacciones que amplían la participación activa y creativa del alumnado con necesidades especiales}

Las AEE como GI y TLD hacen posible no sólo que el alumnado con más dificultades avance y de grandes saltos cualitativos en su aprendizaje gracias a la interacción con los otros. A través de estas actuaciones se consigue también poner de manifiesto lo que este alumnado puede aportar al aprendizaje y progreso del resto del grupo. En ocasiones, porque en la interacción se visibilizan habilidades que este alumnado tiene más desarrolladas, como ocurría en el caso de un alumno con un déficit intelectual importante que sin embargo tenía muy buenas habilidades manipulativas, y en este aspecto podía hacer de modelo y referencia para el resto del grupo. Como la profesora de educación especial nos explicaba:

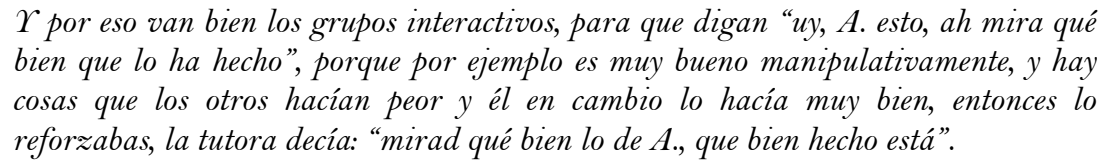

Otras veces, son las propias las interacciones las que han conseguido que el alumnado avance significativamente en las áreas donde tiene más problemas e incluso puede ayudar en ocasiones a sus compañeros. Este tipo de situaciones la ejemplificaba una maestra entrevistada con el caso de un niño con dislexia que, aunque en dificultades importantes en la lectoescritura, fue capaz de resolver mejor que algunos de sus compañeros y compañeras una actividad sobre verbos, y les explicó cómo la había hecho, contribuyendo así a su aprendizaje, de la misma manera que sus compañeros le habían ayudado en otras ocasiones a él.

Este tipo de interacciones se hace posible por el diálogo igualitario que tiene lugar en los GI, en base al cual se hace posible, por ejemplo, que intervengan para ayudar o corregir a un compañero o compañera en aquello que son más competentes, independientemente 
de que sean ellos los que más habitualmente necesitan ayuda y tienen más dificultades; no hay roles preestablecidos que marquen de entrada quien ayuda o quien tiene razón, sino que en cada momento se ponen sobre la mesa todos los conocimientos y los mejores argumentos para que todos progresen en su aprendizaje.

En TLD, la misma niña que aprendió a leer gracias a la tertulia ha progresado cada vez más en su participación en el grupo. Mientras que en un principio era muy callada y estaba muy atenta, pero sin intervenir, poco a poco su capacidad de comprensión lectora ha mejorado, así como su seguridad y confianza, y empezó a hacer más intervenciones. Como relataban la propia niña y su profesora, con el tiempo sus intervenciones han dado lugar a debates muy intensos que sus compañeros y compañeras han seguido con mucho interés, sobre temas como el amor, la fidelidad o la capacidad de decidir de las mujeres. Estos debates no hubieran sido posibles sin la participación de esta niña, por lo que su aportación al grupo es insustituible, y así lo valoran sus compañeros y compañeras, como explica su profesora: "ha hecho intervenciones muy buenas, generando mucho debate, sobre la libertad de decidir (...) Ha hecho que en tertulias sea igual de valorada que los demás". Estos casos nos muestran que, si bien poder observar los avances de este alumnado hace cambiar la imagen y las expectativas hacia ellos y ellas, poder aprender de sus aportaciones es aún más transformador, tanto de su propia autoconfianza como de la imagen que se genera los demás.

\section{Conclusiones}

El análisis de GI y TLD nos muestra que en estas dos Actuaciones Educativas de Éxito tienen lugar interacciones inclusivas para todo el alumnado, incluyendo aquél con más dificultades. Son interacciones generadoras de ayuda que consiguen superar o reducir dificultades en tareas concretas de aprendizaje, avanzar en aprendizajes nuevos superando expectativas basadas únicamente en las capacidades individuales, y hacer aportaciones que crean nuevas oportunidades de aprendizaje para los compañeros y compañeras. Estas mejoras se consiguen dentro del funcionamiento ordinario de estas prácticas, lo que encaja con un enfoque inclusivo, donde se da respuesta a las necesidades de alumnado diverso en un marco común y en relación a unas actividades y contenidos de aprendizaje compartidos (Carrington, 1999). Esto ocurre en unos contextos de aula donde profesorado, especialistas, familiares y otras personas de la comunidad colaboran para diversificar los apoyos al aprendizaje de todo el alumnado, en la línea de una concepción amplia de la idea de apoyo que se ha identificado como necesaria para avanzar hacia la inclusión (Sandoval, Simon y Echeita, 2012). A través de estas actuaciones se evitan los efectos negativos de la segregación y de la diferenciación de contenidos y actividades de aprendizaje, y aparecen oportunidades de aprendizaje que implican un reto cognitivo elevado, y que promueven mayores aprendizajes.

La importancia del contexto en el aprendizaje que vemos reflejada en estas actuaciones contribuye a superar mitos que cuestionan las posibilidades y alcance de la inclusión por centrarse en una perspectiva individual del déficit. Enfoques interactivos como los que se promueven a través de Grupos Interactivos y Tertulias Literarias Dialógicas permiten hablar de un modelo social de la discapacidad, que algunos autores han asociado a la inclusión (Chenoweth y Stehlik, 2004; Nind y Cochrane, 2002). En este modelo la discapacidad no depende única ni principalmente del déficit, sino que es modulada de manera muy importante por el contexto dentro y fuera de la escuela. En las Actuaciones 
Análisis de las Interacciones entre Alumnado y Diversas Personas Adultas en Actuaciones... R. García Carrión, S. Silvia Molina, L. A. Grande y N. Buslón

Educativas de Éxito este contexto está conformado por las interacciones y las oportunidades de diálogo que, en la línea de las actuales teorías del aprendizaje, potencian la elaboración conjunta de conocimiento, la argumentación y el razonamiento (Edwards y Mercer, 1987; Flecha, 1997; Wells, 1999). Estas interacciones y diálogos son lo que permiten transformar condiciones desiguales de partida porque, en palabras de Bruner, nos permiten ir más allá de lo que está establecido, lo que nos viene dado biológica o socialmente, para crear lo que es posible gracias a la cultura, es decir, a la interacción social (Bruner, 2012).

\section{Referencias}

Aguilar, C., Alonso, J., Padrós, M. y Pulido, M. (2010). Lectura dialógica y transformación en las comunidades de aprendizaje. Profesorado. Revista Interuniversitaria de Formación del Profesorado, 67(24), 31-44.

Ainscow, M. (2003). El desarrollo de las escuelas inclusivas. En M. Ainscow (Aut.), El fracaso escolar: una perspectiva internacional (pp. 319-329). Madrid: Alianza Editorial.

Ainscow, M., Booth, T., Dyson, A., Farrell, P., Frankham, J., Gallannaugh, F., ..., Smith, R. (2006). Improving schools, developing inclusion. Londres: Routlegde.

Ainscow, M., Dyson, A., Goldrick, S. y West, M. (2012). Making schools effective for all: rethinking the task. School Leadership y Management, 32(3), 197-213. doi: $10.1080 / 13632434.2012 .669648$

Alexander, R. J. (2008). Towards dialogic teaching: rethinking classroom talk. York: Dialogos.

Alexander, R. J. (2001). Culture and pedagogy: international comparisons in primary education. Oxford: Blackwell.

Anderson, R. C., Chinn, C., Waggoner, M. y Nguyen, K. (1998). Intellectually stimulating story discussions. En J. Osborn y F. Lehr (Eds.), Literacy for all: Issues in teaching and learning (pp. 170-186). Nueva York: Guilford Press.

Aubert, A., Flecha, A., García, C., Flecha, R. y Racionero, S. (2008). Aprendizaje dialógico en la sociedad de la información. Barcelona: Hipatia

Bakhtin, M. M. (1981). The dialogic imagination: four essays by M.M. Bakhtin. Austin, TX: University of Texas Press.

Blatchford, P., Kutnick, P., Baines, E. y Galton, M. (2003). Toward a social pedagogy of classroom group work. International Journal of Educational Research, 39, 153-172. oi: Booth, T. y Ainscow, M. (2002). Guía para la evaluación y mejora de la educación inclusiva. Index for inclusion. Madrid: Consorcio Universitario para la Educación Inclusiva.

Bruner, J. (1996). The culture of education. Cambridge, MA: Harvard University Press.

Bruner, J. (1997). La educación, puerta de la cultura. Madrid: Visor Dis.

Bruner, J. (2012). What psychology should study. International Journal of Educational Psychology, I(1), 5-13.

Carrington, S. (1999). Inclusion needs a different school culture. International Journal of Inclusive Education, 3(3), 257-268. doi:10.1080/136031199285039

Chenoweth, L. y Stehlik, D. (2004). Implications of social capital for the inclusion of people with disabilities and families in community life. International Journal of Inclusive Education, 8(1), 59-72. doi:10.1080/1360311032000139467 
Clark, R. E. y Estes, F. (1998). Technology or craft: what are we doing? Educational Technology, $38(5), 5-11$.

Clark, R. E. y Estes, F. (1999). The development of authentic educational technologies. Educational Technology, 37(2), 5-16.

Cole, M. (1996). Cultural psychology: a once and future discipline. Cambridge, MA: Harvard University Press.

Creswell, J. W. (2002). Educational research: Planning, conducting, and evaluating quantitative and qualitative research. Upper Saddle River, NJ: Pearson Education.

Dunn, L. M. (1968). Special education for the mildly retarded. Is much of it justifiable? Exceptional Children, 35, 5-22.

Durán, D. y Valdebenito, V. (2014). Desarrollo de la competencia lectora a través de la tutoría entre iguales como respuesta a la diversidad del alumnado. Revista Latinoamericana de Inclusión Educativa, 8(2), 141-160.

Edwards, D. y Mercer, N. (1987). Common Knowledge: the development of joint understanding in the classroom. Londres: Methuen.

Estes, F. y Clark, R. E. (1999). Authentic educational technologies: the lynchpin between theory and practice. Educational Technology, 37(6), 5-13.

European Commission. (2011). Communication from the EC. Tackling early school leaving: a key contribution to the Europe 2020 Agenda. Bruselas: European Commission.

European Council. (2010). Council conclusions of 11 May 2010 on the social dimension of education and training. Bruselas: European Council.

Fisher, D., Roach, V. y Frey, N. (2002). Examining the general programmatic benefits of inclusive schools. International Journal of Inclusive Education, 6(1), 63-78. doi:10.1080/13603110010035843

Fitch, F. (2003). Inclusion, Exclusion, and ideology: special education students' changing sense of self. Urban Review, 35(3), 233-252.

Flecha, R. (1997). Compartiendo palabras. El aprendizaje de las personas adultas a través del diálogo. Barcelona: Paidós.

Flecha, R. (2015). Successful educational action for inclusion and social cohesion in Europe. París: Springer Publishing Company.

Flores, L. y Villardón-Gallego, L. (2015) Actitudes hacia la inclusión educativa de futuros maestros de inglés. Revista Latinoamericana de Inclusión Educativa, 9(1), 43-61

Galton, M., Hargreaves, L., Comber, C., Pell, T. y Wall, D. (1999). Inside the primary classroom: 20 years on. Londres: Routledge.

García-Carrión, R. y Díez-Palomar, J. (2015) Learning communities: pathways for educational success and social transformation through interactive groups in mathematics. European Educational Research Journal, 14, 151-166.

Gómez, A., Puigvert, L. y Flecha, R. (2011). Critical communicative methodology: informing real social transformation through research. Qualitative Inquiry, 17(3), 235-245. doi:10.1177/1077800410397802

Habermas, J. (1987). Teoría de la acción comunicativa. Madrid: Taurus.

Herrero, C. y Brown, M. (2010). Distributed cognition in community-based education. Journal of Psychodidactics, 15(2), 253-268. 
Análisis de las Interacciones entre Alumnado y Diversas Personas Adultas en Actuaciones... R. García Carrión, S. Silvia Molina, L. A. Grande y N. Buslón

Howe, C. y Abedin, M. (2013). Classroom dialogue: A systematic review across four decades of research. Cambridge Journal of Education, 43(3), 25-56. doi:10.1080/0305764x.2013.786024

INCLUD-ED. (2011). Actuaciones de éxito en las escuelas europeas. Madrid: Instituto de Formación del Profesorado, Investigación e Innovación Educativa.

Kirschner, P. A., Martens, R. L. y Strijbos, J. W. (2004). CSCL in higher education? A framework for designing multiple collaborative environments. En P. Dillenbourg (Ed.), Computersupported collaborative learning (pp. 3-30). Boston, MA: Kluwer Academic.

López, M., Echeita, G. y Martin, E. (2009). Conceptions on the process of educational inclusion of students with learning disabilities in compulsory secondary education. Cultura y Educación, $21(4), 485-496$.

Mercer, N. (2000). Words and minds: how we use language to think together. Londres: Routledge. doi: $10.4324 / 9780203464984$

Mercer, N. y Littleton, K. (2007). Dialogue and the development of children's thinking: A socio-cultural approach. Londres: Routledge. doi:10.4324/9780203946657

Molina, S. y Ríos, O. (2010). Including students with disabilities in learning communities. Psychology, Society y Education, 2(1), 1-9.

Molina, S. (2015). Alba, a girl who successfully overcomes barriers of intellectual disability through dialogic literary gatherings. Qualitative Inquiry, 21(10), 927-933. doi:10.1177/1077800415611690

Nind, M. y Cochrane, S. (2002). Inclusive curricula? Pupils on the margins of special schools. International Journal of Inclusive Education, 6(2), 185-198. doi:10.1080/13603110110067217

Nystrand, M. (2006). Research on the role of classroom discourse as it affects reading comprehension. Research in the Teaching of English, 40, 392-412.

Nystrand, M. y Gamoran, A. (1991). Instructional discourse, student engagement, and literature achievement. Research in the Teaching of English, 142, 261-290.

Nystrand, M., Gamoran, A. y Heck, M. J. (1993). Using small groups for response to and thinking about literature. The English Journal, 82(1), 14-22. doi:10.2307/820670

Nystrand, M., Gamoran, A., Kachur, R. y Prendergast, C. (1997). Opening dialogue. Nueva York: Teachers College Press.

Oakes, J. (2005). Keeping track: how schools structure inequality. Londres: Yale University Press.

Pea, R. (1993). Practices of distributed intelligence and designs for education. En G. Salomon (Ed.), Distributed cognitions: psychological and educational considerations (pp. 47-88). Nueva York: Cambridge University Press.

Pulido, C. y Zepa, B. (2010). La interpretación interactiva de los textos a través de las tertulias literarias dialógicas. Signos 43(2), 295-309.

Racionero, S. y Padrós, M. (2010). The dialogic turn in educational psychology. Revista de Psicodidáctica, 15(2), 143-162.

Racionero, S., Ortega, S., García, R. y Flecha, R. (2012). Aprendiendo contigo. Barcelona: Hipatia.

Resnick, L. B., Asterhan, C. y Clarke, S. N. (Eds.). (2015). Socializing intelligence through academic talk and dialogue. Washington, DC: American Educational Research Association.

Rogoff, B. (1990). Apprenticeship in thinking: cognitive development in social context. Oxford: Oxford University Press. 
Rojas-Drummond, S. M. y Mercer, N. (2003). Scaffolding the development of effective collaboration and learning. International Journal of Educational Research, 39, (1-2), 99-111. doi.org/10.1016/S0883-0355(03)00075-2

Sandoval, M., Simon, C. y Echeita, G. (2012). Analysis and critical evaluation of support teachers' functions from the standpoint of inclusive education. Revista de Educacion, 1, 117-137.

UNESCO. (1990). World declaration on education for all and framework for action. Jomtien: UNESCO.

UNESCO. (1994). The Salamanca statement and framework for action on special needs education. Adopted by the world conference on special needs education: access and quality. Salamanca: UNESCO.

Valls, R. y Kyriakides, L. (2013). The power of interactive groups: how diversity of adults volunteering in classroom groups can promote inclusion and success for children of vulnerable minority ethnic populations. Cambridge Journal of Education, 43(1), 17-33,

Vygotsky, L. S. (1962). Thought and language. Boston, MA: MIT Press.

Vygotsky, L. S. (1979). El desarrollo de los procesos psicológicos superiores. Barcelona: Crítica.

Wells, G. (1999). Dialogic inquiry: toward a sociocultural practice and theory of education. Boston, MA: Cambridge University Press.

Wenger, E. (2001). Comunidades de práctica: aprendizaje, significado e identidad. Barcelona: Paidós

Wertsch, J., Del Río, P. y Álvarez, A. (1995). Sociocultural studies of mind. Boston, MA: Cambridge University Press.

\section{Breve CV de los autores}

\section{Rocío García Carrión}

Investigadora Bizkaia Talent en la Facultad de Psicología y Educación de la Universidad de Deusto. Como investigadora Marie Curie en la Universidad de Cambridge ha desarrollado su línea de investigación en aprendizaje dialógico, interacción en el aula y el desarrollo intelectual y social del alumnado. Coordinó el trabajo científico del proyecto INCLUD-ED. Strategies for inclusion and social cohesion in Europe from education (FP6, Comisión Europea), ha sido investigadora principal del proyecto Children's Personal Epistemologies: capitalising on children's and families' knowledge for effective learning and teaching (FP7, European Commission). Ha publicado en revistas científicas de impacto científico. Preside la Asociación Multidisciplinar de Investigación Educativa (AMIE) y es miembro del consejo de WERA (World Educational Research Association). Email: rocio.garcia@deusto.es

\section{Silvia Molina Roldán}

Psicóloga y doctora en Pedagogía, profesora en el Departamento de Pedagogía de la Universitat Rovira i Virgili (Tarragona, España) e investigadora del grupo de investigación MEDIS de la Universitat Rovira i Virgili y del centro de investigación CREA de la Universidad de Barcelona. Su principal línea de investigación se centra en la inclusión del alumnado con necesidades especiales en las comunidades de aprendizaje. Ha participado en proyectos de investigación como INCLUD-ED. Strategies for inclusion 
Análisis de las Interacciones entre Alumnado y Diversas Personas Adultas en Actuaciones... R. García Carrión, S. Silvia Molina, L. A. Grande y N. Buslón

and social cohesion in Europe from education (FP6, Comisión Europea), IMPACT-EV. Evaluating the impact and outcomes of EU SSH research (FP7, Comision Europea), y SALEACOM. Overcoming Inequalities in Schools and Learning Communities: Innovative Education for a New Century (H2020, Comisión Europea).Email: silvia.molina@urv.cat

\section{Luis Antonio Grande López}

Maestro de Educación Primaria, Educación Infantil y Audición y Lenguaje y es asesor de Educación Primaria en el Centro de Profesorado de Jerez de la Frontera responsable del área de Comunidades de Aprendizaje. Ha ejercido su función docente fundamentalmente en intervenciones de audición y lenguaje con alumnado con NEE y en Educación Infantil, durante 18 años. También ha coordinado proyectos de innovación educativa sobre la intervención con alumnado con Trastornos del Espectro Autista desde una perspectiva inclusiva, así como un proyecto de investigación educativa sobre el desarrollo de la función simbólica en alumnado con NEE. Email: luis.grande.edu@juntadeandalucia.es

\section{Nataly Buslón Valdez}

Investigadora predoctoral en el Departamento de Teoría Sociológica, Filosofía, Derecho y Metodología de las Ciencias Sociales y en el centro de investigación CREA de la Universidad de Barcelona. Licenciada en Sociología por la Universidad de la República (Uruguay), Máster en Investigación en Sociología y doctoranda en Sociología en la Universidad de Barcelona. Ha sido consultora del Banco Interamericano de Desarrollo (BID) y asistente investigación del Ministerio de Desarrollo Social, Departamento Evaluación y Monitoreo (MIDES) de Uruguay y actualmente es ayudante de investigación en los proyectos IMPACT-EV. Evaluating the impact and outcomes of EU SSH research (FP7, Comisión Europea) y ERASMUS+. Email: nataly.buslon@ub.edu 